\title{
A Candidate Gene Association Study Identifies DAPL1 as a Female-Specific Susceptibility Locus for Age-Related Macular Degeneration (AMD)
}

\author{
Felix Grassmann • Ulrike Friedrich • Sascha Fauser • Tina Schick • \\ Andrea Milenkovic • Heidi L. Schulz • Claudia N. von Strachwitz • \\ Thomas Bettecken • Peter Lichtner • Thomas Meitinger • Nicole Arend • \\ Armin Wolf • Christos Haritoglou • Guenther Rudolph • Usha Chakravarthy • \\ Giuliana Silvestri - Gareth J. McKay - Sandra Freitag-Wolf • Michael Krawczak • \\ R. Theodore Smith • John C. Merriam • Joanna E. Merriam • Rando Allikmets • \\ Iris M. Heid • Bernhard H. F. Weber
}

Received: 28 October 2014/Accepted: 30 January 2015/Published online: 14 February 2015 (C) The Author(s) 2015. This article is published with open access at Springerlink.com

\begin{abstract}
Age-related macular degeneration (AMD) is the leading cause of blindness among white caucasians over the age of 50 years with a prevalence rate expected to increase markedly with an anticipated increase in the life span of the world population. To further expand our knowledge of the genetic architecture of the disease, we pursued a candidate gene approach assessing 25 genes and a total of 109 variants. Of these, synonymous single nucleotide polymorphism (SNP) rs17810398 located in death-
\end{abstract}

Electronic supplementary material The online version of this article (doi:10.1007/s12017-015-8342-1) contains supplementary material, which is available to authorized users.

F. Grassmann · U. Friedrich · A. Milenkovic

H. L. Schulz · B. H. F. Weber $(\bowtie)$

Institute of Human Genetics, University of Regensburg, FranzJosef-Strauss-Allee 11, 93053 Regensburg, Germany

e-mail: bweb@klinik.uni-regensburg.de

S. Fauser · T. Schick

Department of Ophthalmology, University Hospital of Cologne,

53127 Cologne, Germany

C. N. von Strachwitz

EyeCentre Southwest, 70563 Stuttgart, Germany

T. Bettecken

Max Planck Institute of Psychiatry, 80804 Munich, Germany

P. Lichtner · T. Meitinger

Institute of Human Genetics, Helmholtz Zentrum Munich, 85764 Neuherberg, Germany

T. Meitinger

Institute of Human Genetics, Technical University Munich,

81675 Munich, Germany associated protein-like 1 (DAPL1) was found to be associated with AMD in a joint analysis of 3,229 cases and 2,835 controls from five studies [combined $P_{\mathrm{ADJ}}=1.15 \times$ $10^{-6}$, OR 1.332 (1.187-1.496)]. This association was characterized by a highly significant sex difference $\left(P_{\text {diff }}=0.0032\right)$ in that it was clearly confined to females with genome-wide significance $\left[P_{\mathrm{ADJ}}=2.62 \times 10^{-8}\right.$, OR 1.541 (1.324-1.796); males: $P_{\mathrm{ADJ}}=0.382$, OR 1.084 (0.905-1.298)]. By targeted resequencing of risk and nonrisk associated haplotypes in the DAPL1 locus, we identified additional potentially functional risk variants, namely a common 897-bp deletion and a SNP predicted to affect a

N. Arend · A. Wolf · C. Haritoglou · G. Rudolph University Eye Hospital, Ludwig-Maximilians-University, 80336 Munich, Germany

U. Chakravarthy · G. Silvestri

Centre for Experimental Medicine, Queen's University of Belfast, Belfast BT12 6BA, Northern Ireland, UK

\section{G. J. McKay}

Centre for Public Health, Queen's University of Belfast, Belfast BT12 6BA, Northern Ireland, UK

S. Freitag-Wolf · M. Krawczak

Institute of Medical Informatics and Statistics, Christian-

Albrechts University, 24105 Kiel, Germany

R. T. Smith - J. C. Merriam - J. E. Merriam - R. Allikmets Department of Ophthalmology, Columbia University, New York, NY 10032, USA

R. T. Smith

Department of Ophthalmology, New York University School of Medicine, New York, NY 10016, USA 
putative binding site of an exonic splicing enhancer. We show that the risk haplotype correlates with a reduced retinal transcript level of two, less frequent, non-canonical DAPL1 isoforms. DAPL1 plays a role in epithelial differentiation and may be involved in apoptotic processes thereby suggesting a possible novel pathway in AMD pathogenesis.

Keywords Age-related macular degeneration . Death-associated protein-like 1, DAPL1 - Canonical DAPL1 isoforms - Genetic association study

\section{Introduction}

Age-related macular degeneration (AMD) is a common condition of complex etiology with major risk factors including age, gender, smoking, ethnicity and genetics (Zarbin et al. 2014). While AMD ultimately represents the primary cause of blindness in developed countries (Resnikoff et al. 2004), its early form is less severe and characterized by the mere presence of drusen and pigmentary abnormalities in the macular area of the retina (Sarks et al. 1999). Late stage AMD manifests as choroidal neovascularization and/or geographic atrophy and is associated with irreversible central visual loss (Ferris et al. 2005; Zarbin et al. 2014).

Genetic predisposition plays an important role in AMD and is estimated to contribute up to $70 \%$ of the disease risk (Seddon et al. 2005). To date, two major and several minor to moderate AMD susceptibility loci have been identified with per allele odds ratios (OR) ranging from 1.3 to 3.4 (Fritsche et al. 2013). Of note, many of these loci suggest an involvement of inflammatory processes and impaired complement activation in AMD pathogenesis (Klein et al. 2005; Gold et al. 2006; Yates et al. 2007; Hughes et al. 2007; Fagerness et al. 2009), a fact that has raised major interest in novel therapeutic approaches to address progression of the disease (Troutbeck et al. 2012).

Genetic variants associated with complex diseases are usually identified by high-throughput genome-wide association studies of large numbers of cases and controls (Fu et al. 2013). However, candidate gene studies with similar sample sizes normally have greater statistical power to detect genetic disease associations (Amos et al. 2011), especially for genes not covered efficiently by

\section{R. Allikmets}

Department of Pathology and Cell Biology, Columbia University, New York, NY 10032, USA

\section{M. Heid}

Department of Genetic Epidemiology, University of Regensburg, 93053 Regensburg, Germany commercially available genotyping platforms (Wilkening et al. 2009).

In this study, we aimed to expand our current knowledge of the genetic architecture of AMD pathogenesis, following a candidate gene approach. In a well-powered case-control study, we screened 109 haplotype tagging variants in 25 genes for an association with late stage AMD. Attempts to replicate any positive findings in over 4,000 individuals from four previous studies revealed that variation in the death-associated protein-like 1 (DAPL1) gene is significantly associated with AMD. Importantly, this association is restricted to females and the variants of interest correlate with altered transcription levels of specific retinal isoforms of the DAPL1 gene.

\section{Results}

Association of 109 SNPs in 25 Candidate Genes with Late Stage AMD

We first selected 25 genes and 109 haplotype tagging single-nucleotide polymorphisms (SNPs) for an initial analysis of 710 late stage AMD cases and 612 controls (GER1) (Table 1; Supplementary Tables S1, S2). Criteria for candidate gene selection included one or a combination of the following: (1) causative involvement of the gene in phenotypically related retinopathies, (2) known gene function compatible with suspected AMD pathogenesis, (3) specific or predominant gene expression in cellular sites of primary AMD pathology, i.e., the photoreceptor/retinal pigment epithelium (RPE)/choroid complex. All SNPs were tested for significant deviation from Hardy-Weinberg equilibrium $(P<0.05)$ in all controls and in female and male controls separately. This identified three SNPs (RGR:rs2279227, rs4620343 and TRPM3:rs3812532) which were subsequently excluded from further analyses. Association tests adjusted for age and sex revealed a nominally significant association using logistic regression between AMD and three SNPs (DAPL1: rs17810398:C $>\mathrm{T}$, $P=0.016 ; \quad R P 1: \quad$ rs9643828: $>\mathrm{C}, \quad P=0.037 ; \quad C S T 3$ : rs2424577:C $>\mathrm{T}, P=0.028$ ) (Supplementary Table S2).

Replication of Three Nominally Significant AMD-Associated Candidate Gene Variants

The three SNPs with a nominally significant AMD association were genotyped in an independent German replication sample consisting of 996 late stage AMD cases and 645 controls (GER2). The disease association could be confirmed only for rs17810398 $(P=0.0014)$, a synonymous SNP in the coding sequence of the death-associated protein-like 1 (DAPL1) gene (Table 2). Analysis of this 
Table 1 Summary characteristics of participating study populations

\begin{tabular}{|c|c|c|c|c|c|c|c|c|c|c|c|}
\hline \multirow[t]{2}{*}{ Stage } & \multirow[t]{2}{*}{ Study } & \multicolumn{6}{|c|}{ Number of individuals } & \multirow[t]{2}{*}{ Study type } & \multicolumn{3}{|c|}{ Mean age (SD) [years] in } \\
\hline & & Cases & $\mathrm{GA}^{\mathrm{a}}$ & $\mathrm{NV}^{\mathrm{b}}$ & $G A \& N^{c}$ & Controls & Total & & Cases & Controls & Fraction male $(\%)$ \\
\hline 1 & GER1 & 710 & 161 & 423 & 126 & 612 & 1,322 & Case/control & $78.81(6.64)$ & $78.21(5.28)$ & 36.99 \\
\hline 2 & GER2 & 996 & 216 & 535 & 245 & 645 & 1,641 & Case/control & $76.15(7.32)$ & $73.05(8.34)$ & 37.72 \\
\hline 2 & US & 681 & 165 & 516 & 0 & 367 & 1,048 & Case/control & $79.08(8.48)$ & $74.57(7.10)$ & 39.79 \\
\hline 2 & UK & 300 & 38 & 252 & 10 & 183 & 483 & Case/control & $78.45(9.75)$ & $74.53(8.91)$ & 34.78 \\
\hline 2 & $\mathrm{COL}$ & 542 & 55 & 459 & 28 & 1,028 & 1,570 & Population based & $75.49(7.11)$ & $69.51(5.82)$ & 42.93 \\
\hline $1+2$ & ALL & 3,229 & 635 & 2,185 & 409 & 2,835 & 6,064 & Mixed & 77.68 (7.78) & 73.14 (7.49) & 39.36 \\
\hline
\end{tabular}

a Geographic Atrophy

${ }^{\mathrm{b}}$ Neovascular AMD

${ }^{c}$ Individuals with both GA and NV in either the same eye or in different eyes

SNP in three other studies $(681 / 367$ late stage cases/controls from US, 300/183 from UK and 542/1028 from Cologne, Table 1) yielded consistent results (Table 2). Combined analyses of the 3,229 cases and 2,835 controls yielded a $\mathrm{P}$ value of $1.15 \times 10^{-6}$ after adjustment for age, sex and study (Table 2). Given that 106 tests were performed, this result is significant at a significance level of $1.2 \times 10^{-4}$ after Bonferroni correction. The risk allele frequencies were similar in all four studies (13.3-14.3\% in cases; $10.3-12.4 \%$ in controls) and the per allele OR were consistent in direction and magnitude $(1.177 \leq \mathrm{OR} \leq$ 1.530) with no indication for heterogeneity $\left(I^{2}=0\right)$.

Imputation and Replication of Genetic Variants at the DAPL1 Locus

We next imputed the genotypes of 20,422 additional SNPs around rs17810398 in the GER1 study based on 8 tagging SNPs at the DAPL1 locus. After quality control, 517 SNPs were included in the analysis and association signals $\left(P_{\text {ADJ }}<0.05\right)$ were obtained that were confined to a $154-\mathrm{kb}$ region devoid of any gene other than DAPL1 (Fig. 1; Supplementary Table S3). Forty-eight imputed SNPs revealed an association more significant than $\operatorname{rs} 17810398\left(P_{\mathrm{ADJ}}=\right.$ 0.027 ), with a minimum $P_{\mathrm{ADJ}}$ of 0.014 at rs74923781 and its perfect proxy rs17810816: $\mathrm{A}>\mathrm{G}\left(r^{2}=1\right.$ based on 1,000 Genomes CEU samples) in DAPL1 intron 4. De novo genotyping of rs17810816 gave consistent association in terms of its direction in all four replication studies with a nominal significance $\left(P_{\mathrm{ADJ}} \leq 0.05\right)$ attained in GER2 and US. There was no indication of heterogeneity $\left(I^{2}=0\right)$. The combined analysis yielded a $P_{\mathrm{ADJ}}$ of $1.76 \times 10^{-7}$ after adjustment for age, sex and study (Table 2; Fig. 1).Linkage disequilibrium (LD) and haplotype analysis in the GER1 study revealed rs17810398 and rs17810816 to be in moderate LD in controls $\left(r^{2}=0.55\right.$, Supplementary Figure S1).
Variants rs1710398 and rs17810816 Show a FemaleSpecific Association

Stratifying the combined analysis by phenotype, including AMD subtype and age-group, revealed no subgroupspecific association for rs17810398 or rs17810816 (Fig. 2). However, stratification by sex revealed that the association signals of both SNPs were confined to females with genome-wide significance (rs1710398: $P_{A D J}=2.62 \times 10^{-8}$, rs17810816: $\left.P_{A D J}=2.68 \times 10^{-8}\right)$. No AMD association was evident in males (rs1710398: $P_{A D J}=0.382$, rs17810816: $P_{A D J}=0.141$; Fig. 2, Supplementary Figure S2; Table 2). The difference between sex-specific ORs was statistically significant (rs17810398: $P_{\text {diff }}=0.0034$, rs17810816: $P_{\text {diff }}=0.014$ ) at the $5 \%$ level and was observed in all studies analyzed (Supplementary Figure S2; Table 2). In the combined study, the minor allele frequency (MAF) of rs17810398 was lower in female controls than in male controls and higher in female cases than in male cases. A similar, albeit less pronounced effect was seen for variant rs17810816.

\section{DAPL1 Encodes Four Isoforms in Retina/RPE}

For expression analysis of the DAPL1 locus, we scrutinized expressed sequence tags (EST) and identified three entries [GenBank accession numbers: DA417123 (thalamus), BG818506 (oligodendroglioma), BI016096 (lung tumor)] that suggested alternative splicing of DAPll gene products. A potential correlation between rs 1710398 and rs 17810816 genotype and the occurrence of DAPL1 isoforms was investigated by 3 '-RACE experiments on four unrelated RPE/ retina tissue samples, two of which were homozygous (ID_16 and ID_17) for the non-risk alleles and two of which were heterozygous (ID_13 and ID_14) for the risk alleles. After plasmid cloning of PCR products, we sequenced 1,200 cDNA clones and identified a total of 24 specific DAPL1 


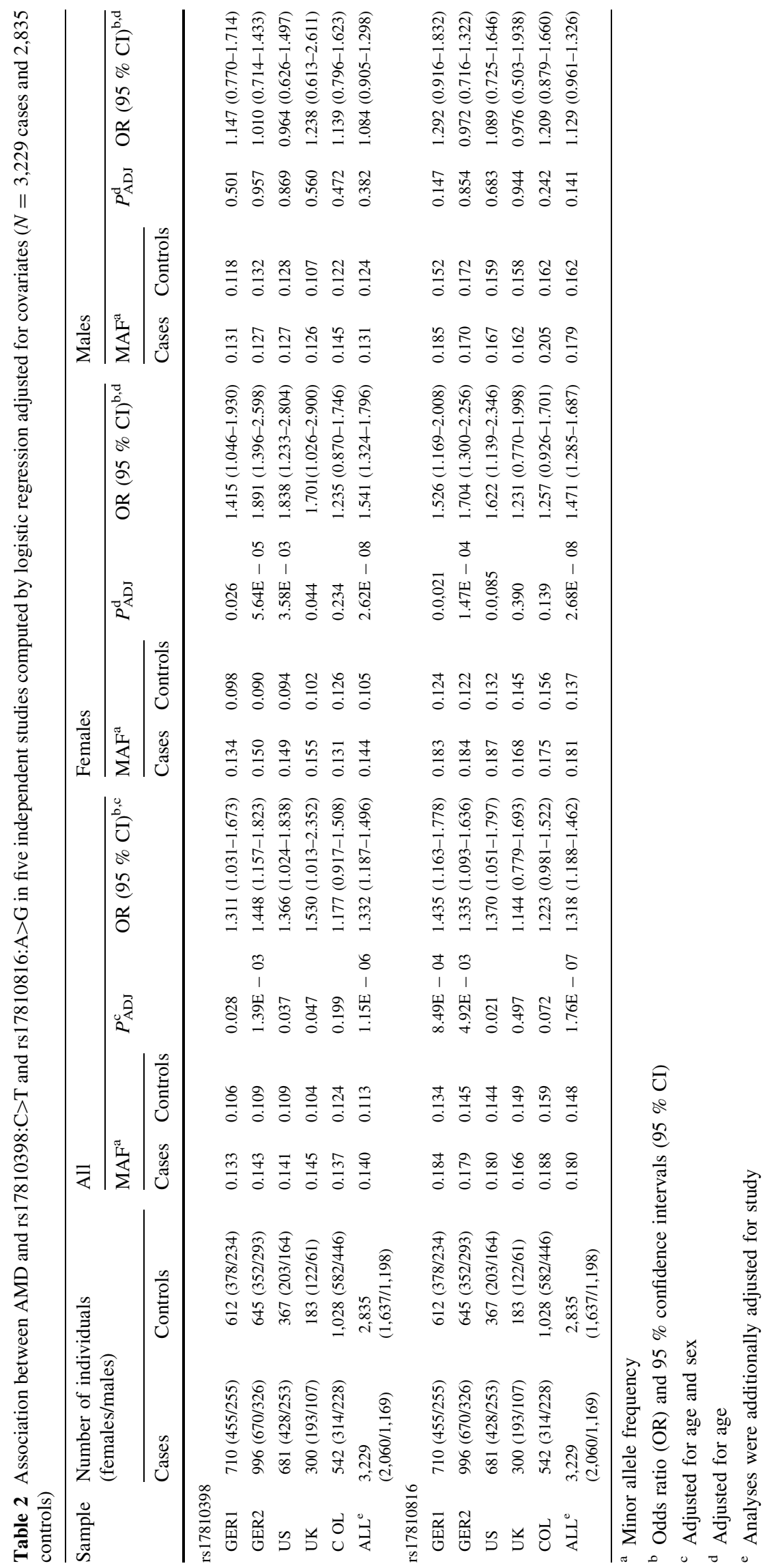


isoforms four of which (referred to as isoforms 1-4, Supplementary Figure S3) were consistently found in all samples. The most abundant isoform 1 (65-77 \% over all samples) corresponded to the DAPLI reference sequence (NM_001017920). Isoform 2 (6-12 \%) and 3 (3-6 \%) had not been reported before, whereas isoform 4 (3-6\%) matched EST BG818506 (Fig. 3a, b). Sequences corresponding to DA417123 and BI016096 were not detected in the RPE/retina RNA samples. RT-PCR analysis confirmed the expression of isoforms 1-4 in human tissues with isoform 4 likely being specific for RPE/retina (Supplementary Figure S4).

Resequencing of Candidate Regions at the DAPL1 Locus

In a search for additional risk variants at the extended DAPL1 locus, we resequenced over $10 \mathrm{~kb}$ of intronic/

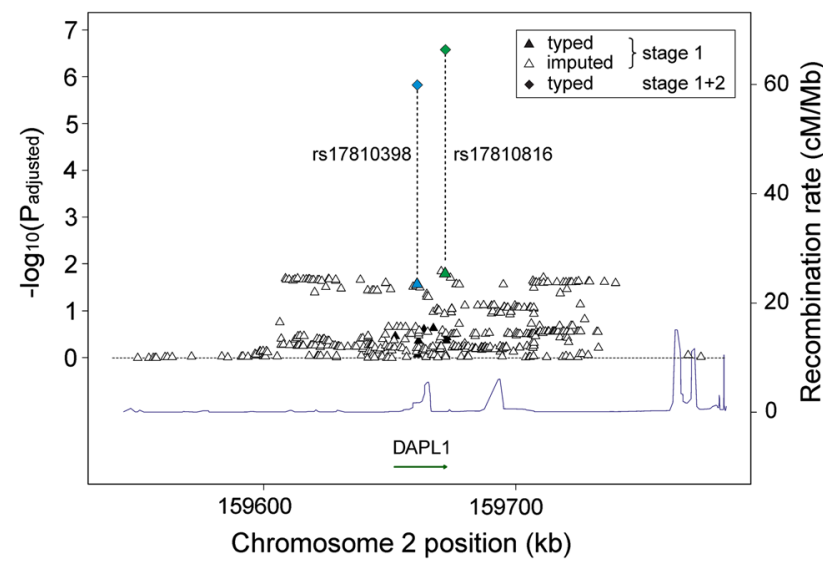

Fig. 1 Association with AMD of imputed and typed variants at the DAPL1 locus. Association signals of markers are shown by the log$P$ value from a logistic regression model (additive model adjusted for age and sex; $y$-axis) and are plotted against their physical position $(x$ axis). Stage 1 results (GER1 sample) are marked by filled (genotyped) and open triangles (imputed). Association signals of rs17810398 and rs17810816 in the pooled samples (3,229 cases and 2,835 controls) are indicated by blue and green diamonds, respectively exonic sequences in each of 12 probands homozygous for AMD risk alleles rs17810398:T and rs17810816:G and eight probands homozygous for AMD non-risk alleles rs17810398:C and rs17810816:A (Supplementary Figure S3; Supplementary Table S4). Due to its extensive saturation with repeat structures, resequencing of the genomic region around DAPL1 exon 4 of HQ179937 (isoform 4) was carried out for three individuals following subcloning of PCR fragments. In total, we detected 33 sequence variants (Supplementary Table S5), three of which (rs75277023:G $>$ A, rs6146986, and rs144087548:A $>$ T) were in strong LD with rs17810398 and rs17810816 $\left(r^{2}\right.$ in controls $>0.9$, Supplementary Figure S1). Variants rs6146986 and rs144087548 were of particular interest as the minor allele of the former represents a common 878-bp deletion in DAPL1 intron 2, and the latter was predicted to affect a putative binding site of an exonic splicing enhancer (serine/arginine-rich splicing factor 1, SRSF1), 24-bp upstream of the most $3^{\prime}$ exon shared by isoforms 3 and 4 (Supplementary Figure S3). Genotyping of rs6146986 and rs 144087548 in the GER1 study confirmed their strong LD with rs17810398 (rs6146986: $r^{2}=0.93$ ) and rs17810816 (rs144087548: $r^{2}=0.77$ ) (Supplementary Figure S1; Table 3). Additional cDNA resequencing of eight RPE/ retina tissues heterozygous for rs17810398 did not reveal additional coding variants (Supplementary Table S6).

AMD-Associated Variants are Correlated with Differential Expression of DAPL1 Isoforms

Samples heterozygous for rs17810398 (ID_13 and ID_14) were characterized by a significantly different abundance of non-risk and risk isoforms 3 and $4\left(P<10^{-4}\right)$. This was not the case for isoforms 1 and 2 (Fig. 3c; Supplementary Table S7). DAPL1 isoform expression in RPE/retina was further evaluated in vivo by semi-quantitative cDNA sequencing (Fig. 4). Of 39 unrelated RPE/retina tissues available, seven were heterozygous for AMD-associated
Fig. 2 Subgroup analysis in the combined study of candidate SNPs rs 17810398 and rs 17810816 in the DAPL1 gene. OR and corresponding $95 \%$ confidence intervals are given with the size of each rectangles representing the respective number of cases. AMD phenotypic subgroups comprise patients with geographic atrophy (GA), and neovascular AMD (NV) and both late stage forms (GA\&NV)

$\begin{array}{lll}\text { Sample } & \text { Cases } & \text { Controls } \\ \text { All } & 3229 & 2825 \\ \text { All }<75 \text { years } & 972 & 1594 \\ \text { All }>75 \text { years } & 2103 & 1068 \\ \text { All male } & 1169 & 1198 \\ \text { All female } & 2060 & 1637 \\ \text { All GA } & 680 & 2837 \\ \text { All NV } & 2192 & 2837 \\ \text { All GA\&NV } & 409 & 2837\end{array}$
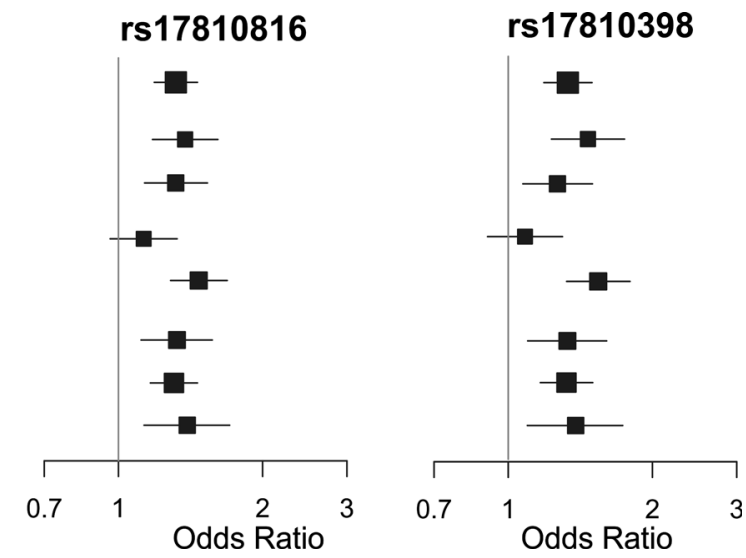
Fig. 3 Functional

consequences for isoform expression of DAPL1 variants. a Exon/intron structure of four frequent DAPL1 isoforms (gene orientation is from left to right). SNP positions are marked by vertical dotted lines.

b Frequency of the four isoforms as determined from sequencing 1,200 cDNA clones that were obtained after $3^{\prime}$ RACE of four unrelated RPE/ retina tissue samples either homozygous (ID_16, ID_17) or heterozygous (ID_13, ID_14) for the non-risk alleles of rs17810398 and rs17810816. c Distribution of rs 17810398 alleles in heterozygous RPE/ retina tissue samples ID_13 and ID_14. Statistically significant deviations from the reference transcript (i.e., isoform 1) are indicated by asterisks $(P<0.0001)$
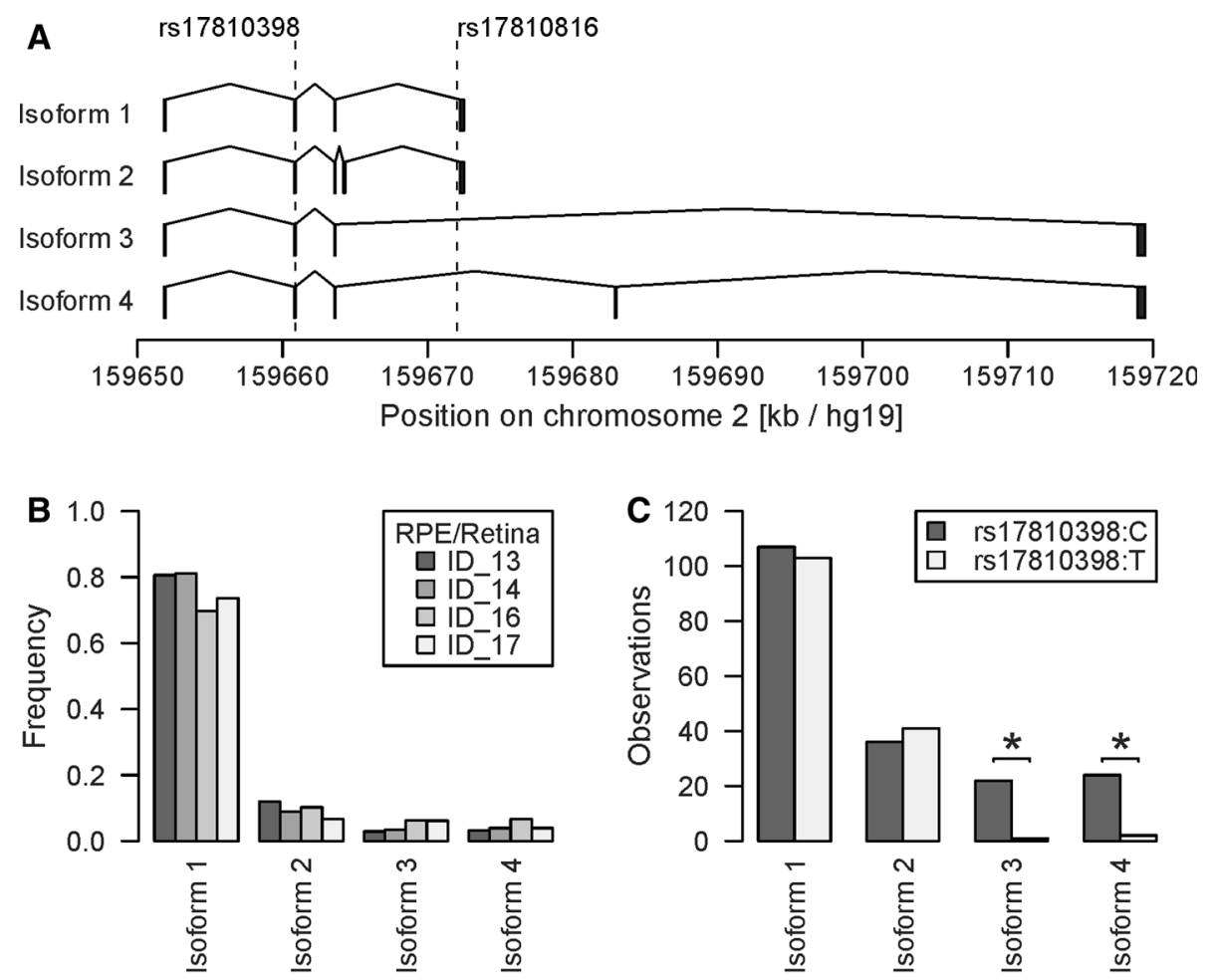

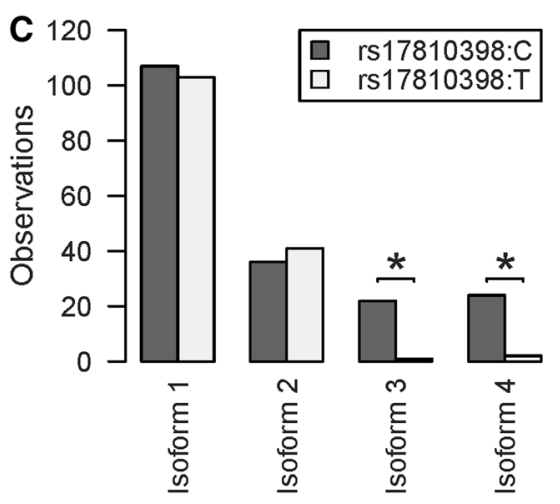

Table 3 Association results in the GER1 study for four functional candidate SNPs in DAPL1

\begin{tabular}{|c|c|c|c|c|c|c|c|c|}
\hline \multirow[t]{2}{*}{ SNP } & \multirow{2}{*}{$\begin{array}{l}\text { Position on chr } \\
2 \text { (bp/hg19) }\end{array}$} & \multirow{2}{*}{$\begin{array}{l}\text { Major } \\
\text { allele }\end{array}$} & \multirow[t]{2}{*}{ Minor allele } & \multicolumn{2}{|l|}{ MAF $^{\mathrm{b}}$} & \multirow[t]{2}{*}{ Odds ratio $(95 \% \mathrm{CI})$} & \multirow[t]{2}{*}{$P^{\mathrm{a}}$} & \multirow[t]{2}{*}{$\mathrm{R}^{2 \mathrm{c}}$} \\
\hline & & & & Cases $(\%)$ & Controls (\%) & & & \\
\hline rs17810398 & $159,660,870$ & $\mathrm{C}$ & $\mathrm{T}$ & 13.3 & 10.5 & $1.314(1.033-1.680)$ & 0.027 & Ref. \\
\hline rs6146986 & $159,661,997-159,662,874$ & - & 878-bp deletion & 13.5 & 10.7 & $1.310(1.033-1.667)$ & 0.027 & NA \\
\hline rs17810816 & $159,671,992$ & A & G & 18.4 & 13.4 & $1.435(1.163-1.778)$ & $8.49 \times 10^{-4}$ & 0.866 \\
\hline rs144087548 & $159,718,894$ & A & $\mathrm{T}$ & 17.4 & 13.8 & $1.296(1.047-1.609)$ & 0.018 & 0.735 \\
\hline
\end{tabular}

${ }^{\mathrm{a}} P$ from logistic regression adjusted for age and sex

b MAF: minor allele frequency calculated in 710 cases or 612 controls

${ }^{\mathrm{c}} \mathrm{R} 2$ to top variant based on CEU samples from the 1,000 genomes project

variants rs17810398, rs6146986, rs17810816, and rs144087548. In agreement with our 3'-RACE data, these samples revealed differential expression of isoforms 3 and 4 but not isoforms 1 and 2 (Fig. 4). Interestingly, sample ID_11 was heterozygous for rs17810398, but homozygous for the non-risk alleles of rs6146986, rs17810816, and rs144087548. In this sample, expression intensities of isoform 3 and 4 alleles were equal excluding rs17810398 as a functional variant involved in the differential expression of isoforms 3 and 4. This leaves rs6146986, rs17810816, and rs144087548 or an as yet unknown but correlated variant as the truly functional risk variant at the DAPL1 locus.

\section{Discussion}

Here, we provide evidence that DAPL1 is an AMD-associated gene and that its disease association is female-specific. To our knowledge, this is a first study reporting a sexspecific genetic association with AMD at a genome-wide significance level. Although lead SNPs rs17810398 and rs17810816 have been imputed into large GWAS data sets, neither variant has been identified before as AMD-associated (Fritsche et al. 2013). This is likely due to the female specificity of the association as male/female ratios in multicenter GWAS tend to differ greatly between cohorts thereby potentially leading to reduced power. Behrens et al. 


\begin{tabular}{|c|c|c|c|c|c|c|c|c|}
\hline RPE/retina & ID_A & ID_B & ID_C & ID_E & ID_2 & ID_11 & ID_13 & ID_14 \\
\hline rs17810398 & $\mathrm{C} / \mathrm{T}$ & $\mathrm{C} / \mathrm{T}$ & $\mathrm{C} / \mathrm{T}$ & $\mathrm{C} / \mathrm{T}$ & $\mathrm{C} / \mathrm{T}$ & $\mathrm{C} / \mathrm{T}$ & $\mathrm{C} / \mathrm{T}$ & $\mathrm{C} / \mathrm{T}$ \\
\hline rs6146986 & $-/ d e l$ & $-/$ del & $-/$ del & $-/$ del & $-/ \mathrm{del}$ & $-1-$ & $-/ d e l$ & $-/ d e l$ \\
\hline rs17810816 & $A / G$ & $A / G$ & $A / G$ & $A / G$ & $A / G$ & $\mathrm{~A} / \mathrm{A}$ & $A / G$ & $A / G$ \\
\hline rs144087548 & $A / T$ & $A / T$ & $A / T$ & $A / T$ & $A / T$ & $\mathrm{~A} / \mathrm{A}$ & $A / T$ & $\mathrm{~A} / \mathrm{T}$ \\
\hline Isoform $1 \& 2$ & & & & & & & $1 \mathrm{~h}$ & \\
\hline Isoform 3 & & & & & & & & \\
\hline Isoform 4 & & & & & & & & \\
\hline
\end{tabular}

Fig. 4 Semi-quantitative cDNA sequencing of eight RPE/retina tissue samples heterozygous for synonymous coding SNP rs 17810398: $\mathrm{C}>\mathrm{T}$. The chromatograms of the variant nucleotide at rs 17810398 flanked by \pm 3 bp are shown for isoforms $1 \& 2,3$, and 4 . Isoforms were specifically amplified by three different exon-spanning

(2011) have methodologically shown that gender-stratified analyses greatly increase power to detect gender-specific effects. Additionally, we have also observed the femalespecific association in our population-based sample (COL study) after adjusting the analysis for age. This further indicates that different study types increase the heterogeneity and therefore may lead to decreased power to detect this association.

We also considered the possibility that age is a confounding factor in our study since (1) females are slightly older than males and (2) cases are older than controls. If any of the variants would be correlated with longevity, this could potentially confound our analysis. However, we found no evidence for a correlation between either SNP or age, neither in cases, controls, females nor males separately or analyzed jointly $(P>0.05)$. Additionally, we note that logistic regression analyses were adjusted for age in our analyses. Furthermore, two SNPs at the DAPL1 locus (rs9869 and rs10497199) were investigated in a recent study by Flachsbart et al. 2010. For these two variants, the authors found no association with longevity $(P>0.5)$. Variant rs9869 is weakly linked to markers rs17810398 $\left(r^{2}=0.13\right)$ and $\mathrm{rs} 17810816\left(r^{2}=0.1\right)$, while rs10497199 is independent of either variant $\left(r^{2}<0.1\right)$. Taken together, these findings have led us to exclude a confounding effect of age in our analysis.

The observed association in the present study could eventually be explained by a population substructure in our cases or controls from the UK or US. Although we cannot definitely exclude such a possibility, it is of note that primer combinations. Genotypes of the four candidate variants are given above the chromatograms. The sample heterozygous for rs17810398 but homozygous for the non-risk alleles of rs6416986, rs17810816, and rs144087548 is highlighted in red

frequency and effect sizes of the two DAPL1 risk variants rs17810398 and rs17810816 (in males, females and jointly) observed in the UK and US study are similar to the frequencies observed in the combined German cohort (Table 2). The German samples derive from a genetically homogenous population from a small area in southern Germany. Homogeneity was estimated previously from genome-wide data available for a subset of cases (Fritsche et al. 2013). From these data, we conclude that the US and UK study primarily consists of Caucasians which are genetically similar to the German cohort and, if at all, population substructure may only exert a minor effect on study outcome.

Although DAPL1 is evolutionarily conserved, only little is known about its function. It has been shown to be abundantly expressed in the retina/RPE transcriptome (Schulz et al. 2004) as well as in epidermis, esophageal epithelium, and tongue epithelium where it appears to be involved in the early stages of stratified epithelial differentiation (Sun et al. 2006). Based upon strong amino acid sequence similarities, DAPL1 has also been connected to the death-associated protein (DAP), a basic, proline-rich protein of $15-\mathrm{kD}$ molecular weight that acts as a positive mediator of programmed cell death upon induction by interferon-gamma (Deiss et al. 1995). Clarification of the cellular function of DAPL1 in the RPE/retina is required to allow more detailed insight into this novel pathway of AMD pathogenesis.

We have shown that DAPL1 is present in a multitude of correctly spliced isoforms, two of which, isoforms 3 and 4 , 
were specifically down-regulated in the presence of AMDassociated alleles. Although we could not identify the causative variant at the DAPL1 locus, we excluded lead SNP rs17810398 as the presence of the T-risk allele in one patient (ID_11) had no influence on the transcript levels of isoform 3 or 4 . Notably, the unique C-terminus of isoform 4 encodes two potential transmembrane domains with significant homology to the rhodopsin-like $G$ protein-coupled receptor (GPCR) family. Another member of the GPCR family, the G protein-coupled estrogen receptor 1 (GPER) plays a role in intracellular signaling following estrogen binding and could provide a useful lead when searching for factors involved in sex-dependent AMD risk. While at present we cannot explain the gender-specificity of the association with DAPL1, our results provide a starting point at a molecular level to investigate why AMD is more frequent in women than in men (Owen et al. 2012).

Taken together, we investigated 25 gene loci of interest to AMD pathology and excluded all but one from being disease-associated. Our data implicate DAPL1 as a novel gene involved in AMD pathology although the cellular functions of this gene and of its various differentially spliced transcripts remain elusive. Our study revealed a correlation between risk variants at rs17810398 and rs17810816 on the one hand and expression levels of DAPL1 isoforms 3 and 4 on the other, the latter being specifically expressed in RPE/retina tissue. We also reported a significant sex difference of the effect of DAPLI where only females showed an association signal at this locus. Although speculative at present, this sex difference may be explained by a role of DAPL1 variants in sexspecific signaling processes. Our findings add another piece to the puzzle of the genetic architecture of AMD, which, once completed, should allow refined identification of individuals at risk for this disease.

\section{Methods}

\section{Subjects}

Five independent studies were included in our study comprising a total of 3,053 unrelated Caucasian patients with clinically documented late stage AMD (cases) and 2,738 unrelated age and individuals with comparable age range and ethnicity without signs of macular disease (controls) (Table 1). All data were available for analysis at the analysis center in Regensburg. Discovery study GER1 (stage 1) included 710 AMD patients and 612 controls from the University Eye Clinic of Würzburg (Germany). The four replication studies (Stage 2) comprised (1) 996 AMD patients and 645 controls from the University Eye Clinics in München, Tübingen and Würzburg (Germany)
(GER2); (2) 681 AMD patients and 367 controls from Columbia University (New York, USA) (US); (3) 300 AMD patients and 183 controls from the Royal Victoria Hospital (Belfast, UK) (UK); and (4) 542 AMD patients and 1,028 controls from the Department of Ophthalmology at the University Hospital Cologne, Germany (COL). Cases and controls were examined by trained ophthalmologists. Stereo fundus photographs were graded according to standardized classification systems as described previously (Grassmann et al. 2012). The study was conducted at all sites in strict adherence to the tenets of the Declaration of Helsinki and was approved by the respective Ethics Committees at the University Eye Clinics of Würzburg, München and Tübingen, by the Institutional Review Board at Columbia University, by the Research Ethics Committee of Queen's University Belfast and by the local Ethics Committee in Cologne.

\section{Genotyping}

Genomic DNA was extracted from peripheral blood leukocytes according to established protocols. Genotyping of SNPs was carried out by direct sequencing, TaqMan SNP genotyping (Applied Biosystems, Foster City, USA) or by primer extension of multiplex PCR products and subsequent allele detection by matrix-assisted laser desorption/ionization time of flight (MALDI-TOF; Sequenom, San Diego, USA). Direct sequencing was performed with the Big Dye Terminator Cycle sequencing kit version 1.1 (Applied Biosystems, Foster City, U.S.A.) according to the manufacturer's instructions. Reactions were analyzed with an ABI Prism 3130xl sequencer (Applied Biosystems). TaqMan pre-designed SNP genotyping assays (Applied Biosystems) were used according to the manufacturer's instructions. The rs144087548 variant was genotyped by polymerase chain reaction (forward primer: $5^{\prime}$-CGC AGA CAT GAT GCT GGG GGT-3'; reverse primer: 5'-ACA TGC AAG ACG GGG AAT TGA-3') followed by HpyCH4III digestion (New England Biolabs, Ipswich, USA) and restriction fragment length analysis. All SNPs showed high genotyping quality with an average call rate $>98 \%$ in each of the five case-control samples.

Statistical Methods

\section{Discovery Study}

We excluded three SNPs [rs2279227 (RGR), rs4620343 and rs3812532 (TRPM3)], each with significant deviation from Hardy-Weinberg equilibrium (HWE, $P \leq 0.05$ ) in the control group of the discovery sample. SNP association analysis was carried out by logistic regression adjusted for age and sex. All analyses modeled an additive genetic 
effect and the genotype was coded as the number of alleles present at a given variant (i.e., 0, 1 or 2).

\section{Replication Studies and Combined Analysis}

All SNPs were in HWE $(P>0.05)$. We used the same tests for SNP association analysis as in the discovery study. We also combined the individual data from all five studies and also adjusted the respective analyses by study center (coded as factors). The $I^{2}$ measure was computed to measure between-study heterogeneity. We also conducted sex-stratified analyses for each study separately and for all study samples combined. Sex differences were asses for statistical significance using a $t$ test derived from sex-specific beta estimates and corresponding standard errors.

All reported $P$ values were two-sided except where noted otherwise. All SNP association analyses were carried out with R (v3.0.1, http://R-Forge.R-project.org/). To allow a more detailed inspection of the genomic region of interest, measures of LD were calculated using $\mathrm{R}$ package snp.plotter (Luna and Nicodemus 2007).

\section{Imputation of SNPs}

Prior to imputation, 8 tag SNPs in DAPL1 were phased in the GER1 study individuals using SHAPEIT2 (Delaneau et al. 2013). Then, untyped SNPs were imputed with IMPUTE2 (Howie et al. 2009) using the 1,000 Genomes Phase I integrated haplotypes (release 20110521) as reference panel. After the exclusion of SNPs with imputation quality ("info") $<0.5$, the genotype probabilities (dosages) of the remaining SNPs were also analyzed by logistic regression in R, using an additive model adjusted for age and sex.

\section{Genomic Resequencing}

Genomic resequencing was done for regions of interest defined by the presence of certain gene elements (putative promoter, coding exons of transcripts NM_001017920.2, HQ179935, HQ179936, and HQ179937) or conserved elements based upon the "46-Way Most Cons" track of the UCSC genome browser, NCBI Build 37/hg19. Regions within extensive repeat structures were excluded (Supplementary Figure S3). Resequencing primers are listed in Supplementary Table S4.

\section{Prediction of Functional Impact of Risk Variants}

The functional impact AMD-associated SNPs (with known dbSNP ID) on RNA processing as well as protein sequence, structure and function was predicted using the web-based "SNP Function Prediction" tool implemented in the "SNPinfo Web Server" (http://snpinfo.niehs.nih. gov/index.html) (Xu and Taylor 2009). For newly identified SNPs, we used ESEfinder 3.0 to predict the effect of a given SNP allele on putative exonic splicing enhancers (http://rulai.cshl.edu/cgi-bin/tools/ESE3/esefinder.cgi) (Cartegni et al. 2003).

Characterization of Major Splice Variants of DAPL1 in Human Retina/RPE

To determine major splice variants and functional polyadenylation sites, $3^{\prime}$ rapid amplification of cDNA ends ( $3^{\prime}$-RACE) experiments were conducted. RNAs from RPE/ retina tissues that were either heterozygous (ID_13 and ID_14) or homozygous (ID_16 and ID_17) for the non-risk rs17810398:C allele were isolated by RNeasy Mini Kit followed by DNAse I treatment (QIAGEN, Hilden, Germany). 3'-RACE was conducted with the FirstChoice RLM-RACE Kit (Applied Biosystems/Ambion, Austin, USA) according to the manufacturer's instructions. Forward primers for first and second (nested) PCR were 5'-GCA CTG GCA CACG CTA TG-3' and 5'-TTG GCA CCT TGG AAA GAC ATA CC-3', respectively. Amplified RACE products were ligated into the pGEM-T vector (Promega, Madison, USA). PCR products were obtained with M13 forward and M13 reverse primers from a total of 1,200 clones. Of these, 597 clones were sequenced; the remaining 603 could unequivocally be assigned to DAPL1 isoform 1 (NM_001017920.2, HQ179934) by visual gel inspection. The sequences of isoforms 2-6 were submitted to GenBank (HQ179935, HQ179936, HQ179937, HQ179938, HQ179939).

Expression Analysis and Semi-quantitative

Resequencing

Eight RPE/retina tissues with risk variant genotypes as given in Fig. 3 and Supplementary Table S6 were used as templates to amplify isoform-specific PCR products with forward primer 5'-GCA CTG GCA CAC GCT ATG-3' and the isoform-specific reverse primers 5'-CGA GGC TGC TGA ATA ATG TAG-3' (isoform $1 \& 2$ ), 5'-TCT GGA TCC TCT GAG CTT CTT CTC-3' (isoform 3) or $5^{\prime}$-CTG GAT CCT CTG AGC TTC TTG TGT-3' (isoform 4), followed by sequencing with the forward primer. Primers for the GUSB gene were 5'-ACT ATC GCC ATC AAC AAC ACA CTC ACC-3' and 5'-GTG ACG GTG ATG TCA TCG AT- $3^{\prime}$. For tissue samples, sex was determined with fluorescence-based PCR analysis of the homologous, $\mathrm{X}$ - and Y-linked genes AMELX and AMELY as described in Sullivan et al. (1993).

Acknowledgments We thank all patients and control individuals for their participation in the study, Kerstin Meier and Jürgen Kaschkötö (Institute of Human Genetics, University of Regensburg) 
for technical support, and Lars G. Fritsche (Department of Biostatistics, University of Michigan School of Public Health, Ann Arbor, Michigan) and Thomas Winkler (Institute of Epidemiology, University of Regensburg, Regensburg) for help with computational tools and data analysis. This study was supported partly by the Deutsche Forschungsgemeinschaft (WE 1259/19-1 and WE 1259/192 to BHFW), the Alcon Research Institute (to BHFW and RA), by grants from the National Eye Institute/NIH EY013435 and EY019007 (to RA); the Macula Vision Research Foundation (to RA); an unrestricted grant to the Department of Ophthalmology, Columbia University, from Research to Prevent Blindness, Inc. (to RA), and the Guide Dogs for the Blind Association UK (2008-5a) Macular Disease Society (to US, GS, GJM).

Conflict of interest The authors declare no competing financial interests.

Open Access This article is distributed under the terms of the Creative Commons Attribution License which permits any use, distribution, and reproduction in any medium, provided the original author(s) and the source are credited.

\section{References}

Amos, W., Driscoll, E., \& Hoffman, J. I. (2011). Candidate genes versus genome-wide associations: Which are better for detecting genetic susceptibility to infectious disease? Proceedings Biological Sciences/The Royal Society, 278(1709), 1183-1188.

Behrens, G., et al. (2011). To stratify or not to stratify: Power considerations for population-based genome-wide association studies of quantitative traits. Genetic Epidemiology, 35(8), $867-879$.

Cartegni, L., et al. (2003). ESEfinder: A web resource to identify exonic splicing enhancers. Nucleic Acids Research, 31(13), 3568-3571.

Deiss, L. P., et al. (1995). Identification of a novel serine/threonine kinase and a novel $15-\mathrm{kD}$ protein as potential mediators of the gamma interferon-induced cell death. Genes and Development, 9(1), 15-30.

Delaneau, O., Zagury, J.-F., \& Marchini, J. (2013). Improved wholechromosome phasing for disease and population genetic studies. Nature Methods, 10(1), 5-6.

Fagerness, J. A., et al. (2009). Variation near complement factor I is associated with risk of advanced AMD. European Journal of Human Genetics : EJHG, 17(1), 100-104.

Ferris, F. L., et al. (2005). A simplified severity scale for age-related macular degeneration: AREDS Report No. 18. Archives of Ophthalmology, 123(11), 1570-1574.

Flachsbart, F., et al. (2010). Investigation of genetic susceptibility factors for human longevity-A targeted nonsynonymous SNP study. Mutation Research, 694(1-2), 13-19.

Fritsche, L. G., et al. (2013). Seven new loci associated with agerelated macular degeneration. Nature Genetics, 45(4), 433-439.

Fu, W., O'Connor, T. D., \& Akey, J. M. (2013). Genetic architecture of quantitative traits and complex diseases. Current Opinion in Genetics and Development, 23(6), 678-883.
Gold, B., et al. (2006). Variation in factor B (BF) and complement component $2(\mathrm{C} 2)$ genes is associated with age-related macular degeneration. Nature Genetics, 38(4), 458-462.

Grassmann, F., et al. (2012). Modelling the genetic risk in age-related macular degeneration. PLoS ONE, 7(5), e37979.

Howie, B. N., Donnelly, P., \& Marchini, J. (2009). A flexible and accurate genotype imputation method for the next generation of genome-wide association studies. PLoS Genetics, 5(6), e1000529.

Hughes, A. E., et al. (2007). A common CFH haplotype, with deletion of CFHR1 and CFHR3, is associated with lower risk of agerelated macular degeneration. Nature Genetics, 38(10), 1173-1178.

Klein, R. J., et al. (2005). Complement factor $\mathrm{H}$ polymorphism in agerelated macular degeneration. Science, 308(5720), 385-389.

Luna, A., \& Nicodemus, K. K. (2007). snp.plotter: An R-based SNP/ haplotype association and linkage disequilibrium plotting package. Bioinformatics, 23(6), 774-776.

Owen, C. G., et al. (2012). The estimated prevalence and incidence of late stage age related macular degeneration in the UK. The British Journal of Ophthalmology, 96(5), 752-756.

Resnikoff, S., et al. (2004). Global data on visual impairment in the year 2002. Bulletin of the World Health Organization, 82(11), 844-851.

Sarks, S. H., et al. (1999). Early drusen formation in the normal and aging eye and their relation to age related maculopathy: a clinicopathological study. The British Journal of Ophthalmology, 83(3), 358-368.

Schulz, H. L., et al. (2004). The Retinome-Defining a reference transcriptome of the adult mammalian retina/retinal pigment epithelium. BMC Genomics, 5(1), 50.

Seddon, J. M., et al. (2005). The US twin study of age-related macular degeneration: Relative roles of genetic and environmental influences. Archives of Ophthalmology, 123(3), 321-327.

Sullivan, K. M., et al. (1993). A rapid and quantitative DNA sex test: Fluorescence-based PCR analysis of X-Y homologous gene amelogenin. BioTechniques, 15(4), 636-638.

Sun, L., et al. (2006). EEDA: A protein associated with an early stage of stratified epithelial differentiation. Journal of Cellular Physiology, 206(1), 103-111.

Troutbeck, R., Al-Qureshi, S., \& Guymer, R. H. (2012). Therapeutic targeting of the complement system in age-related macular degeneration: A review. Clinical and Experimental Ophthalmology, 40(1), 18-26.

Wilkening, S., et al. (2009). Is there still a need for candidate gene approaches in the era of genome-wide association studies? Genomics, 93(5), 415-419.

$\mathrm{Xu}$, Z., \& Taylor, J. A. (2009). SNPinfo: Integrating GWAS and candidate gene information into functional SNP selection for genetic association studies. Nucleic Acids Research, 37(Web Server issue), W600-W605.

Yates, J. R. W., et al. (2007). Complement C3 variant and the risk of age-related macular degeneration. The New England Journal of Medicine, 357(6), 553-561.

Zarbin, M. A., Casaroli-Marano, R. P., \& Rosenfeld, P. J. (2014). Age-related macular degeneration: Clinical findings, histopathology and imaging techniques. Developments in Ophthalmology, 53, 1-32. 